\title{
Design and analysis of different models of microfluidic devices evaluated in Enhanced Oil Recovery (EOR) assays
}

\author{
Gustavo Rosero ${ }^{1}$, Ana Peñaherrera ${ }^{1}$, Carol Olmos ${ }^{1}$, Alejandro Boschan ${ }^{3}$, \\ Pablo Granel $^{2}$, Federico Golmar ${ }^{2}$, Carlos Lasorsa ${ }^{1}$, Betiana Lerner ${ }^{1,4}$, \\ Maximiliano Perez ${ }^{1,4}$
}

\footnotetext{
${ }^{1}$ Universidad Tecnológica Nacional (UTN), Facultad Regional Haedo Grupo de Ingeniería de Recubrimientos Especiales y Nanotecnologia (IREN) Buenos Aires, Paris 532.1076, Argentina.

e-mail: gustavorosero@gmail.com, anibelenp@hotmail.com, carolmaritza1@gmail.com, betianalerner@gmail.com, lasorsa@cnea.gov.ar, max@fullgen.com.ar.

${ }^{2}$ Instituto Nacional de Tecnología Industrial (INTI), Laboratorio de Nanodispositivos INTI-Micro y Nanoelectrónica

Av. Gral. Paz 5445, Buenos Aires, Argentina.

e-mail: pgranell@inti.gob.ar, fgolmar@inti.gob.ar

${ }^{3}$ Universidad de Buenos Aires (UBA), Facultad de Ingeniería (FIUBA)Buenos Aires, Grupo de Medios Porosos

C1063ACV, Argentina.

e-mail: aboschfi@gmail.com.

${ }^{4}$ Universidad de Buenos Aires (UBA), Facultad de Ingeniería (FIUBA) Instituto de Ing. Biomédica C1063ACV, Buenos Aires, Argentina.
}

\begin{abstract}
Microfluidic devices are a new platform for Enhanced Oil Recovery (EOR) assays. The application of Colloidad Dispersion Gels (CDG) injection method has been proposed in recent years. However, the mechanism of oil recovery enhancement has not been completely elucidated. Its purpose is to mobilize oil by decreasing the permeability of channeled zones. The success of injection depends on the characteristics of water, the porous medium and the oil. Therefore, a successful injection in a reservoir can be different in another, hence the importance of a methodology for assessment prior to injection. This work applies micro and nanotechnology techniques to develop microfluidic chips used in EOR tests. EOR chips simulate the phenomena that occur in micro-nano scale reservoirs. Experiments analyzed multiphase flows in porous media inside chips. Generally, the first step of the experiments corresponds to fill the microchannels with water and then oil (1 poral volume 1PV). Second, water-injection takes place at constant flow rate until oil recovery ceases. Finally, either polymer or CDG is injected and their behavior is studied by digital image analysis. Results allowed obtaining graphs of oil residual saturation versus time for pore volumes of fluid used in EOR. The optimum configurations of the microchannels showed $80 \%$ of residual oil saturation after water injection.
\end{abstract}

Keywords: EOR, poral volume, microgels and polymers, microfuidic devices.

\section{INTRODUCTION}

The ability to reduce the amount of oil that remains in a particular well becomes increasingly important as global oil supplies decrease. Methods for recovering oil will be the subject of much scientific and engineering research for many years to come [1]. There are three stages for oil recovery: primary depletion, secondary recovery process and tertiary or enhanced oil recovery. Between the 50 and $70 \%$ of the original oil remains in the oil field after primary and secondary oil recovery techniques have been exhausted [2]. Consequently, there is huge interest in recovering the remaining-trapped oil in the reservoir. The techniques used range from improved secondary flooding method to enhanced methods. The distinction between the classifications of the methods usually refers to the target oil that the process seeks to recover. Then, in improved recovery the aim is to increase the oil sweep efficiency, whereas in tertiary recovery the aim is to mobilize and recover residual of capillary trapped oil [1]. The tertiary oil recovery has various approaches that are chemical, thermal and gas. Moreover, a combination of the last two has been proposed recently Multi 
Thermal Fluid Assisted Gravity Drainage (MFAGD) [3].

Enhanced Oil Recovery (EOR) methods focus on trapped oil mobilization either locally by capillary forces and/or by large scale bypassing during water flooding [4]. In the later stage of water flooding, the water displacement efficiency and economic outcome of water flooding decrease due to reservoir geological and fluid characters. Viscosity difference between the oil and injected water result in viscous fingering towards producers [5]. A dilute aqueous solution of partially hydrolyzed polyacrylamide is used as a pushing fluid in the injection wells to sweep oil in the reservoir into the production well [6]. Other approach to improve EOR is decreasing the mobility ratio (M):

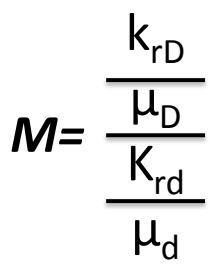

Where $\mathrm{k}_{\mathrm{rD}}$ and $\mu_{\mathrm{D}}$ are relative permeability and viscosity of the displacing phase, respectively, and $\mathrm{k}_{\mathrm{rd}}$ and $\mu_{\mathrm{d}}$ represent the displaced phase [7]. Mobility ratios less than 1 indicate positive displacement. Foam has been used to lower mobility ratio as it diminishes mobility by reducing gas relative permeability $\mathrm{k}_{\mathrm{rD}}$ and by increasing the effective viscosity $\mu_{\mathrm{D}}[7,8]$.

Polymer flooding and colloidal dispersion gels (CDG) have been purposed to increase the sweep efficiency. CDG are defined as an aqueous solution with a polymer concentration from 100 to $1200 \mathrm{ppm}$ and a polymer: cross linker relation from 20:1 to 100:1 [9]. CDG are so named from the nature of the gel solutions, which are suspensions of individual bundles of cross-linked polymer molecules, or colloids. A solution of separate gel "bundles" are formed in which a mixture of predominantly intramolecular and minimal intermolecular crosslinking reactions. By contrast, in "bulk gels" the crosslinking mechanisms form a continuous intermolecular network of polymer molecules [10]. CDG are used as a sweep improvement agent in EOR process for displacing the oil where the mobility ratio is unfavorable [10, 11].

The main challenge with chemical methods is that every possible variable related to fluid and substrate properties can change from an oil reservoir to the next one. Hence, the chemistry must be tailored for each reservoir and the oil properties can vary as well. Besides, chemical approach faces another challenge, which is cost, depending on the fluctuating price of oil and production [2].

The mechanism through which a particular EOR method is evaluated depends on reducing residual oil saturation and precisely how that residual oil is trapped at the pore-scale. Pore network modeling has been purposed to achieve this goal, as micromodels are microfluidic devices with transparent walls that enable visualization of fluids within spatially structured pore networks produced by etching or other microfabrication techniques. The flow experiments applying micromodels have been considered a straightforward and powerful experimental method to characterize the two-phase or multiphase flow mechanism, wettability alteration, and pore-scale oil recovery performance $[3,4,12,13]$.

As there are distinct oil fields, it becomes impossible to perform in situ measurements. Therefore, experiments uses core samples of the actual oil field or similar artificial made oils to test the ability of penetrating fluids to either displace the oil from pressure flow or imbibitions, which is where the displacing agent wets the substrate by either natural wettability or by modification process. This method does not represent a model to analyze the process at the pore and capillary level [2]. Conversely, micro-models have also been used to investigate specific aspects related to flow in porous media [14], capillary pressure [15] and interfacial tension [16]. KAMARI, et al. [17] applied glass micro-models to obtain quantitatively measurements as well as qualitatively investigations of fractured and non-fractured porous media [17]. 3D models have also been proposed to compare Steam Assisted Gravity Drainage (SAGD) with MFAGD [3].

Micro-devices can offer an alternative to simulate physical and chemical properties of sandstone. NILSSON, et al. [2] studied the impact of different rheological properties of the driving fluids applying a series of microfluidic devices tailored to precisely reproduce a two-dimensional slice from a sandstone core.

Microfluidics has been defined as the study and manipulation of fluids in micrometer scale structures [18]. It has been widely used in several applications, and the interest in the oil industry is increasing. The labon-chip system pursues integrating laboratory work into a chip platform [19]. A 2-D polydimethylsiloxane (PDMS) microfluidic device has been applied to analyze oil displacement mechanisms and foam mobility. 
PDMS allows to design a system that simulates the high and low permeability zones stratified in the direction of the injection [7].

In this study, microfluidics is applied to simulate oil displacement by water as baseline for oil recovery; polymer and CDG flooding are applied within microchannels with geometry that simulates the porosity of oil reservoirs. This paper aims to find a microchip configuration that traps between 30 and $80 \%$ oil after water injection. The micromodels with different architecture are used as a model to perform fluid injections and to extend the understanding of oil displacement through water, polymer and CDG flooding to evaluate their behavior when exposed to capillary entry pressures of the matrix regions of the microchannels.

\section{MATERIALS AND METHODS}

\subsection{Design and fabrication of the microfluidic device}

Three-microfluidic devices have been designed using layout editor software (http://www.layouteditor.net). In the first chip, labeled "E", there are three different permeability and porosity zones (low-medium-high) where architecture involves three different sizes of regular octagons with repeated separations between them, to generate the distinct permeabilities. High permeability zone consists in 28 structures of $2 \mathrm{~mm}$, with total area of $235 \mathrm{~mm}^{2}$. Medium permeability zone has 55 structures of $1 \mathrm{~mm}$ with total area of $218 \mathrm{~mm}^{2}$. Low permeability zone has 109 structures of $0.5 \mathrm{~mm}$ with total area of $100 \mathrm{~mm}^{2}$ as described in Figure $1 \mathrm{~A}$. Second chip "AD0" has been divided in two zones with two types of octagons to create zones with different permeability. $85 \mathrm{~mm}^{2}$ is the total area of AD0 chip, which contains 714 structures, 680 of $0.25 \mathrm{~mm}^{2}$, and 34 de $1 \mathrm{~mm}^{2}$, as described in Figure 1B. Fluids only occupied the area previously mentioned and calculated. Finally, chip "AB0" contains two different zones, with two different sizes of squares and constant separations between them, to produce two types of permeabilities and porosity into whole chip. It has 275 structures with a total area of $85 \mathrm{~mm}^{2}, 70$ of $0.25 \mathrm{~mm}^{2}$, and 205 of $1 \mathrm{~mm}^{2}$, as described in Figure 1C.

Table 1 details the characteristics of the micromodels designs that were used.

Table 1: Summary of micromodels features.

\begin{tabular}{c|c|c|c|c|c}
\hline CHIP & PERMEABILITY & $\begin{array}{c}\text { POROSITY } \\
\%\end{array}$ & $\begin{array}{c}\text { PORAL VOLUME } \\
(\mu \mathrm{L})\end{array}$ & $\begin{array}{c}\text { HEIGHT } \\
(\mu \mathrm{m})\end{array}$ & CHARACTERISTICS \\
\hline \multirow{3}{*}{ "E" } & High & 76 & $\approx 180$ & \multirow{2}{*}{180} & 3 permeabilities \\
\cline { 2 - 4 } & Medium & 65 & $\approx 140$ & & 2 permeabilities \\
\cline { 2 - 5 } "AD0" & Low & 46 & $\approx 5$ & 180 & \\
OCTAGONS & High & 61 & $\approx 50$ & 180 & 2 permeabilities \\
\hline "AB0" & Heterogeneous & 59 & $\approx 54$ & & \\
\hline
\end{tabular}

Microfluidic devices were fabricated in polydimetilsiloxane (PDMS) casted from a mold. PDMS replicates the features of the master mold with high fidelity (tens of nanometers) [20]. The desired designs are molded in high relief by a prototyping technique such as photolithography in a silicon wafer $700 \mu \mathrm{m}$ thick (Virginia Semiconductor, Inc.), with the negative resin SU-8 (MicroChem). The silicon substrate was cleaned by sonication with acetone and isopropyl alcohol, and substrate surface was dehydrated for 10 min at $200{ }^{\circ} \mathrm{C}$. Then, SU-8 resist was dispensed on the substrate and spun in two cycles. The spinner was accelerated for $5 \mathrm{~s}$ at $100 \mathrm{rpm} \cdot \mathrm{s}^{-1}$ until $500 \mathrm{rpm}$, and held at $500 \mathrm{rpm}$ for $5 \mathrm{~s}$. In the spin cycle, a ramp of $300 \mathrm{rpm} \cdot \mathrm{s}^{-1}$ was applied until $2000 \mathrm{rpm}$, and held for $30 \mathrm{~s}$. The resist was soft baked firstly at $65{ }^{\circ} \mathrm{C}$ for $20 \mathrm{~min}$, and secondly at $95{ }^{\circ} \mathrm{C}$ for $50 \mathrm{~min}$. The substrate was aligned and the resist was exposed to near UV at $650 \mathrm{~mJ}$. The first step of a post-exposure bake consisted on $65^{\circ} \mathrm{C}$ for $12 \mathrm{~min}$, and the second step was performed at $95{ }^{\circ} \mathrm{C}$ for $15 \mathrm{~min}$. Finally, the resin was developed for $15 \mathrm{~min}$ under agitation. SU-8 mold over a silicon wafer was fabricated inside cleanroom facilities. PDMS chip fabrication steps are described in supplementary material (APPENDIX 1). 


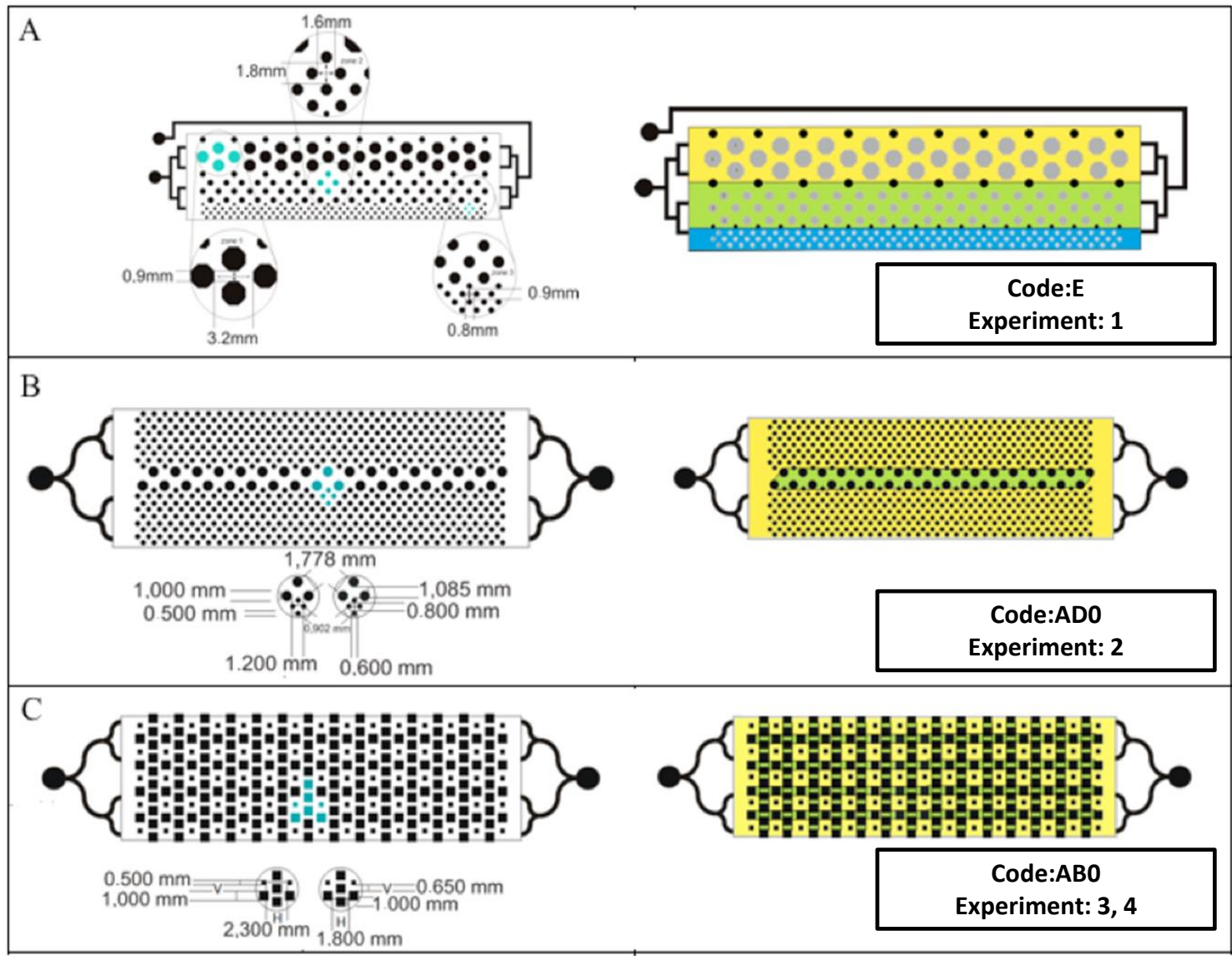

Figure 1: Architecture of (A) chip "E" where lower permeability zone is depicted in blue, intermediate permeability zone is colored in green and high permeability zone is indicated in yellow, (B) chip "AD0" where high permeability zone is colored in green and low permeability zone is depicted in yellow, $(\mathrm{C})$ chip "AB0" where low permeability zone is colored in green whereas high permeability zone is indicated in yellow. Images are not in scale.

\subsection{Experimental Procedures}

Four experiments were performed in which three chip designs and different recovery injection fluids were used. EOR experiments were performed at room temperature. Commercially available, partially hydrolyzed polyacrylamide (HPAM) (which will be referred as polymer) was used for experiment 1 . In contrast, CDG mixture with crosslinker (20:1) was applied during experiment 2, whereas, CDG mixture without crosslinker was employed for experiment 3 and 4. Ultrapure water was used in all experiments. In order to perform digital image analysis, water was stained with blue dye (acid blue 22 C.I. 42755). The physicochemical properties of the oil used are $4.42 \mathrm{cP}$ and $0.8105 \mathrm{gcm}^{-3}$ at room temperature. The images of residual oil distribution after water, polymer and CDG injection covered the entire porous zone. The oil residual saturation and poral volume were determined via image processing of recorded video. Each pixel was assumed to represent a single-phase fluid volume. Cameras used were Guppy PRO F-125B / C resolution of 1.2 MP with Nikon lens of $28 \mathrm{~mm}$ and Coolsnap CF 12 bits with Nikon lens of $28 \mathrm{~mm}$. Image analysis was made by image processing of recorded video through FIJI software with the assumption of a 2-D fluid system.

\section{RESULTS}

Experiments were divided into three stages: A, B and C as depicted in Figure 2. Each stage represents different fluid injection cases at distinct flooding rates, which are summarized in Table 2. 
A

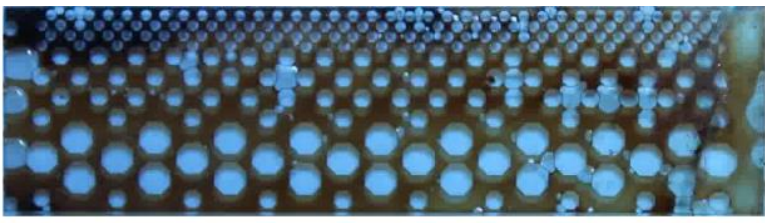

$\mathrm{B}$
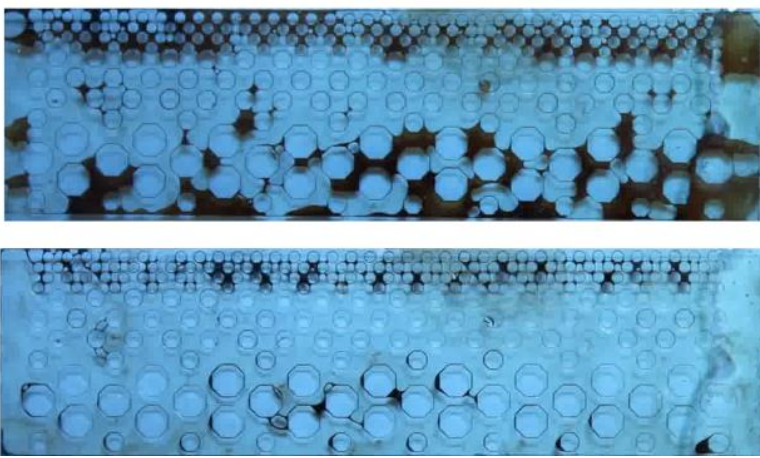

Figure 2: Sequential images of stages A, B and C of experiment with chip "E".

Table 2: Flooding rates of fluids used in the experiments.

\begin{tabular}{c|c|c|c|c}
\hline FLUIDIC & $\begin{array}{c}\text { CHIP E } \\
(\text { EXPERIMENT 1) } \\
\left(\mathrm{mL} \cdot \mathrm{min}^{-1}\right)\end{array}$ & $\begin{array}{c}\text { CHIP ADO } \\
(\text { EXPERIMENT 2) } \\
\left(\mathrm{mL} \cdot \mathrm{min}^{-1}\right)\end{array}$ & $\begin{array}{c}\text { CHIP AB0 } \\
\text { (EXPERIMENT 3) } \\
\left(\mathrm{mL}^{-1} \mathrm{~min}^{-1}\right)\end{array}$ & $\begin{array}{c}\text { CHIP ABO } \\
\text { (EXPERIMENT 4) } \\
\left.\left(\mathrm{mL}^{-m_{i}}\right)^{-1}\right)\end{array}$ \\
\hline Ethanol & - & - & - & 0.5 \\
\hline Water & - & 5 & 0.5 & 3 \\
\hline Oil & 3.6 & 5 & 3 & 3 \\
\hline Water & 5 & 5 & 3 & 3 \\
\hline Fluid & 3.6 & 5 & 5 & 5 \\
\hline
\end{tabular}

1Polymer

2CDG mixture with crosslinker

3CDG mixture without crosslinker

4CDG mixture without crosslinker

1-4(Experiment number)

During waterflooding of a water-wet reservoir, water advances across the porous medium in a fairly flat front [21]. Despite a fairly uniform water front on a macro-scale, a considerable oil fraction is trapped due to entrapment mechanisms on the micro-scale [22]. Figure 3 shows the results obtained from experiment performed with chip E. The images obtained were analyzed with image processing software to determine the areas of each phase. From this analysis, the saturation of the pore network is obtained. Stage A shows water injection case (1.8 PV) that reduces the residual oil saturation to $32 \%$ while stage B depicts polymer injection case $(0.7 \mathrm{PV})$ that decreased the residual oil saturation to $5.4 \%$ approximately. In the water injection case as presented in stage B of Figure 2 the aqueous phase goes across the intermediate permeability zone, it forms a preferential flow path and shows the low macroscopic sweep efficiency. In contrast, in the polymer injection case (stage $\mathrm{C}$ of Figure 2), the higher sweep efficiency can be observed, implying the aqueous-phase preferential flow can be eliminated in polymer injection cases. In the polymer swept zone, the microscopic residual oil saturation is low, and the small blobs of oil prefer to occupy the throats instead of pore bodies. Some disconnected water blobs and oil ganglia could also be observed. The disconnected water blobs have small size and occupy the narrow throat. The isolated oil ganglia have various sizes. These ganglia are often disconnected and thus immobilized by small aqueous phase blobs occupying single throats, which are the possible results of snap-off events. Once trapped by the aqueous phase occupying throats, the oil ganglia are very difficult to re-mobilize due to the driving force that is needed to overcome the capillary entrance pressure of the throat. 


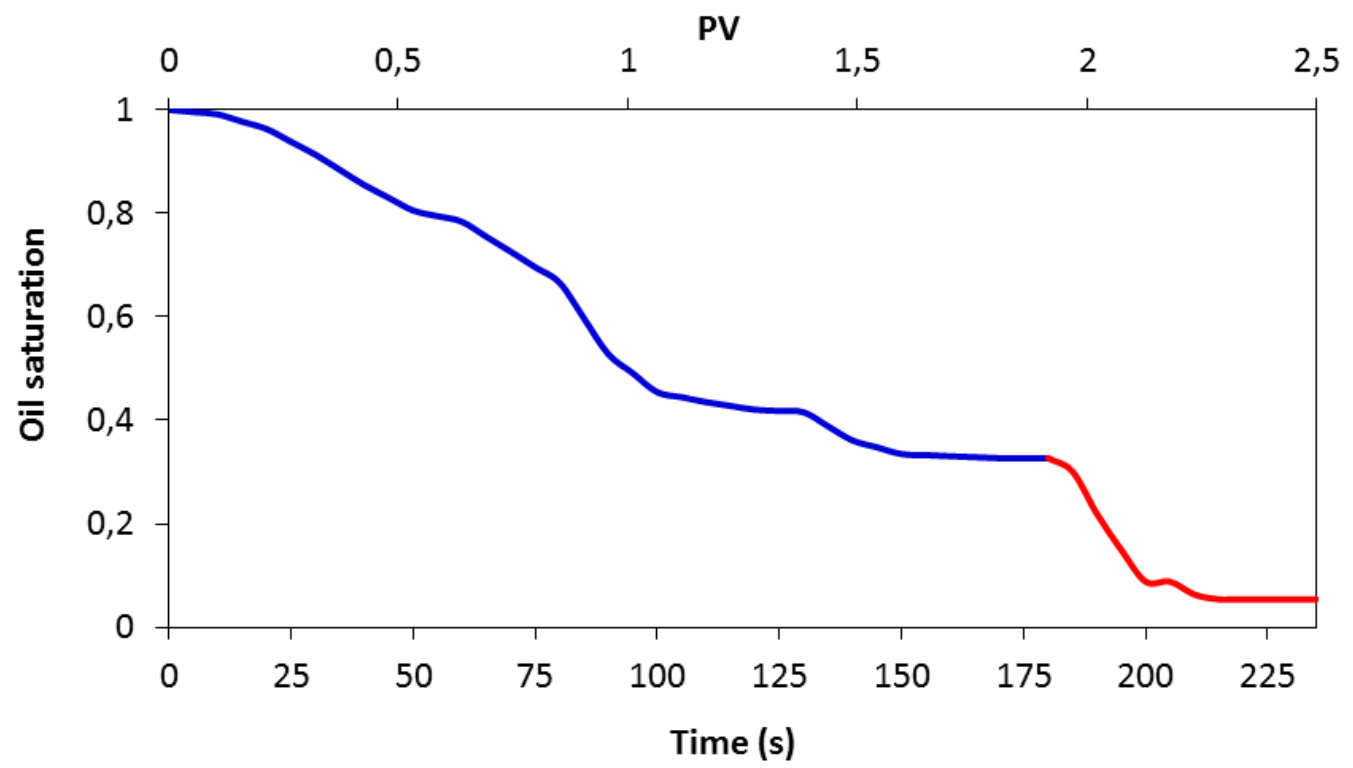

Figure 3: Residual oil saturation (inferior $X$ axis), poral volumes of injected fluids (superior $X$ axis) vs time. Blue line: stage A and Red line: stage B. Stage A shows water injection (1.8 PV) that reduces the residual oil saturation to $32 \%$ while stage B depicts polymer injection ( $0.7 \mathrm{PV})$ that decreased the residual oil saturation to $5.4 \%$.

Experiment 2 allowed the observation of water injection thin finger-like path. This experiment was conducted with chip AD0 and it is described in Figure 4 that presents stages A and B, which corresponds to water injection case (3.3 PV) and CDG mixture with crosslink agent injection case ( $0.67 \mathrm{PV})$, respectively. Water flooding reduced the residual oil saturation to $32.1 \%$ whereas CDG mixture with crosslinker decreased the residual oil saturation to $22.9 \%$. The enhanced properties of CDG mixture with crosslinker are particularly useful in heterogeneous formation, where non-uniform pores results in an irregular flood front and incomplete volumetric sweep [10].

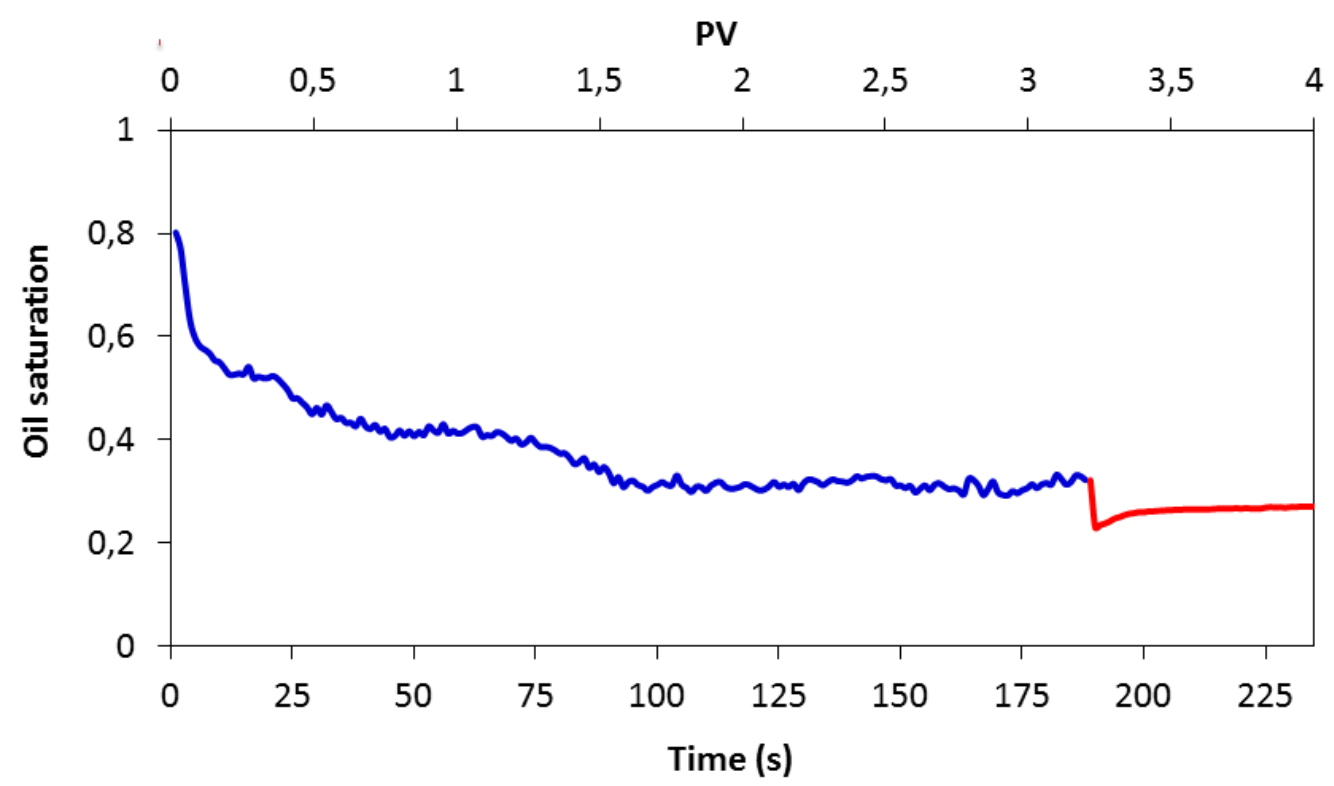

Figure 4: Residual oil saturation (inferior $X$ axis), poral volumes of injected fluids (superior $X$ axis) vs time. Blue line: atage A and Red line: stage B. Stage A corresponds to water injection (3.3 PV) and stage B representes CDG mixture with crosslinking agent injection $(0.67 \mathrm{PV})$. Water flooding reduced the residual oil saturation to $32.1 \%$ whereas $\mathrm{CDG}$ mixture with crosslinker decreased the residual oil saturation to $22.9 \%$. 
Experiments number 3 and 4 were performed with chip AB0 (Figures 5 and 6). These experiments were used to evaluate the reproducibility of oil recovery, the effect of a prolonged injection of water and the effect on the oil recovery of CDG fluid without crosslinker. Bubble formation inside microchannels was avoided through previous ethanol flushing before water injection as superficial tension of ethanol is $(22.3$ $\mathrm{mNm}^{-1}, 20{ }^{\circ} \mathrm{C}$ ) lower than superficial tension of water $\left(72.8 \mathrm{mN} \mathrm{m}^{-1}, 20{ }^{\circ} \mathrm{C}\right)$ [23]. Therefore, the intermolecular forces of hydrogen bonds in ethanol are lower than the forcers in hydrogen bonds in water.

Experiment 3 is described in figure 5. Stage A shows water injection case $0.3 \mathrm{PV}$ and stage B indicates CDG mixture without crosslinking agent injection case $0.67 \mathrm{PV}$. Water injection case extracted $21 \%$ of oil whereas CDG fluid without cross-linker swept $4 \%$ of oil.

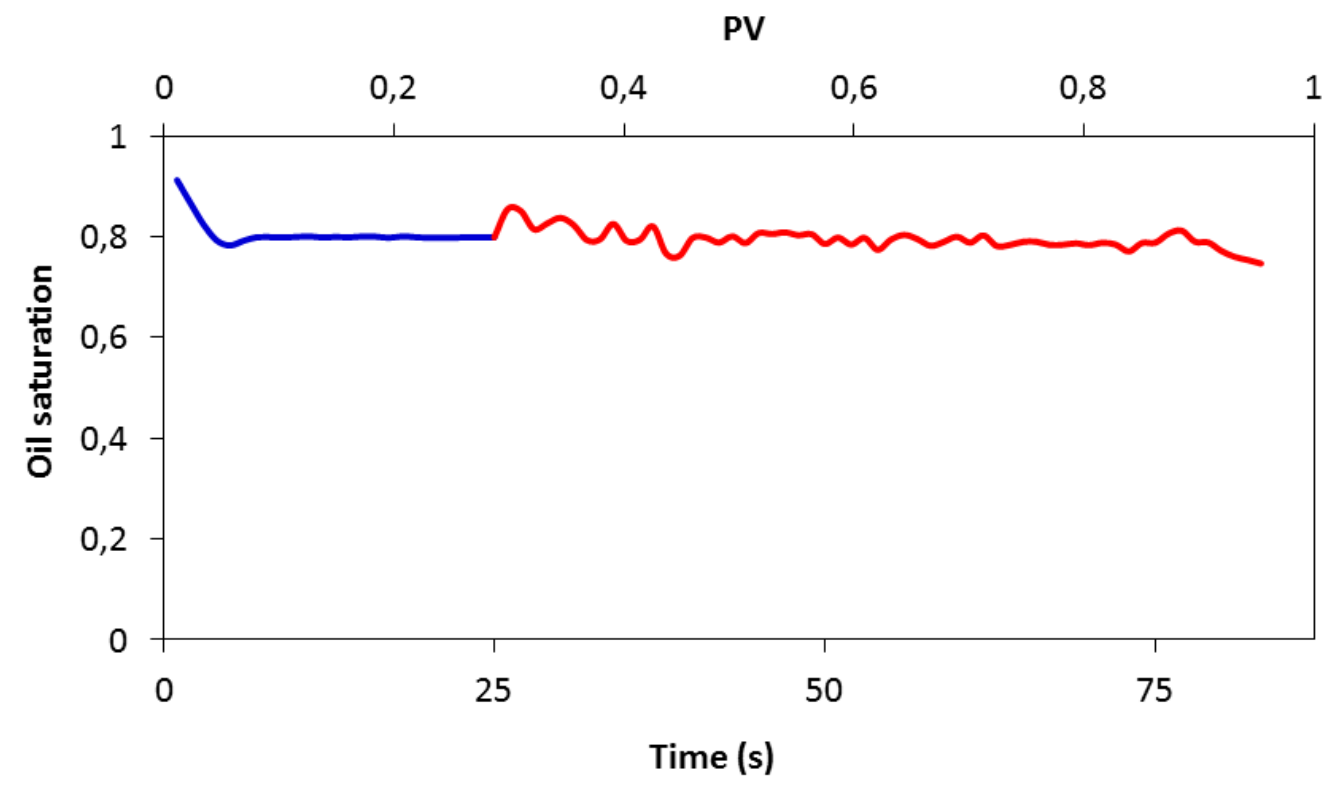

Figure 5: Residual oil saturation (inferior $X$ axis), poral volumes of injected fluids (superior $X$ axis) vs time. Blue line: atage A and Red line: stage B. Stage A shows water injection (0.3 PV) and stage B indicates CDG mixture without crosslinking agente injection (0.67 PV).

Figure 6 presents the results of experiment number 4. Stage A describes water injection case (1 PV), whereas stage B depicts CDG mixture without crosslinking agent injection case. 1PV of this fluid was supplied at $5 \mathrm{~mL} \mathrm{~min}{ }^{-1}$ flow rate. However, the residual oil saturation maintained constant.

For both experiments was observed that CDG mixture without crosslinker was incapable of reducing the residual oil saturation that remained after water flooding as the viscosity was too low to overcome the capillary forces. Furthermore, a prolonged injection of water does not produce an improvement over the oil recovery. CDG without crosslinker had limited propagation across the porous media. On the other hand, the reproducibility of the oil recovery experiments was checked in AB0 micromodel.

It was determined that CDG mixture with crosslinker agent decreased the residual oil saturation effectively compared to CDG mixture without crosslinker agent, implying significant potential oil recovery enhancement [10]. 


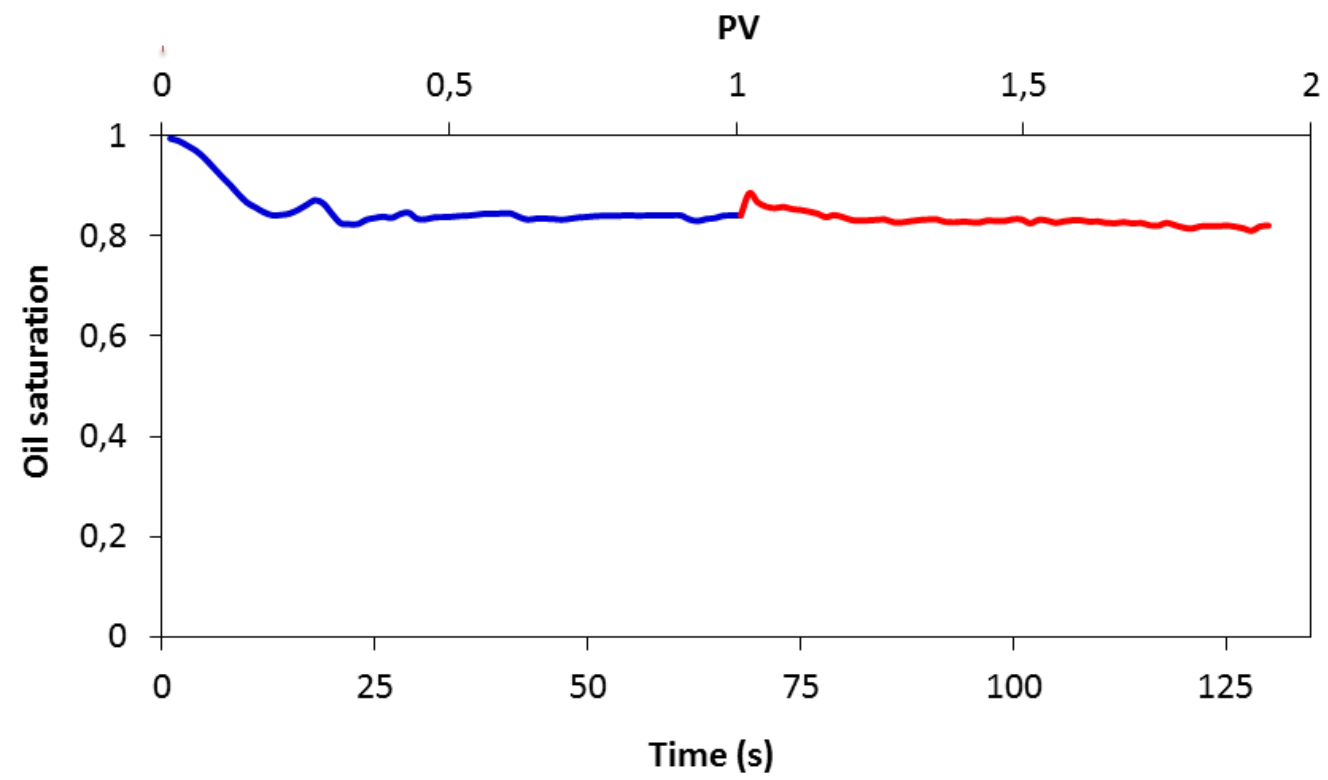

Figure 6: Residual oil saturation (inferior $\mathrm{X}$ axis), poral volumes of injected fluids (superior $\mathrm{X}$ axis) vs time. Blue line: stage A and Red line: stage B. Stage A shows water injection (1 PV) and stage B indicates CDG mixture without crosslinking agente injection (0.9 PV).

\section{DISCUSSION}

Three different architectures were used; their porosities and consequently distinct poral volumes (PV) are detailed in Table 1. The image analysis allowed the measurement of poral volumes of each fluid according to the correspondent gray scale.

Chip E allowed the observation of the oil saturation (1 VP), subsequent displacement produced by the water injection case (1.8 PV) and polymer injection case $(0.7 \mathrm{PV})$ through three different permeabilities. The mechanisms by which water can displace oil is described by three main processes; piston-like displacement, pore body filling and snap-off [24]. Experiment with chip E presents oil entrapment by a snap-off mechanism where water as the wetting phase can advance as surface films while oil still remains in the center of large pores [25]. Wide pore is connected through very small throats; water films in the throats frequently coalesce and cut off globules of oil form the continuous oil phase. Since oil is the non-wetting phase, the large pressure required to overcome the capillary pressure and move the oil globules through the narrow throats effectively traps the disconnected oil in the large pores [21]. After the water front passes almost all the remaining oil is immobile. Due to this immobilization in the water-wet case there is little or no oil production after water breakthrough at the production well, and waterflooding can be stopped [26]. Oil ceases shortly after water breakthrough, which occurred close at two pore volumes of injected water.

After water injection case, the residual oil saturation was $32 \%$ and the preferential path that water goes across corresponds to the intermediate permeability. Therefore, disconnected oil ganglia are observed at the lowest and highest permeabilities. Then, polymer injection case takes place and residual oil saturation gradually decreases from 32 to $5.4 \%$ as polymer advances. This is attributed to viscosity and chemical properties of the polymer, which diminishes the mobility ratio implying a significant improvement in oil sweeping efficiency [1]. In going from water to polymer flooding, the viscous forces are usually considered to be augmented by up to one order of magnitude, which is not thought to be enough to mobilize residual oil. Consequently, the target for polymer flooding is considered to be any oil that is bypassed in the water injection case but does not include residual oil.

Chip AD0 shows two permeabilities zones, which were saturated with oil (1 PV), water injection case (3.3 PV) showed $67.9 \%$ of oil recovery. Oil is left behind in a water flood either because it is trapped by capillary forces (residual oil) or due to it is in some way bypassed. Residual oil is constituted by disconnected oil ganglia, which are immobilized as a result of capillary forces. To mobilize this oil, it is needed to increase greatly the viscous-to-capillary force balance between the water and oil phases in the displacement [1]. Finally CDG fluid with cross-linker was applied $(0.67 \mathrm{PV})$ and residual oil saturation decreased progressively from 32.1 to $22.9 \%$. 
Chip AB0 shows high and low permeabilieties. This chip was used to perform two experiments. First AB0 chip was flushed with ethanol to prevent bubble formation. Then, water was injected to extract ethanol residues. After that, AB0 chip was saturated with oil (1 PV). Water injection case ( $0.3 \mathrm{PV})$ extracted $21 \%$ of oil whereas CDG fluid without cross-linker $(0.67 \mathrm{PV})$ swept $4 \%$ of oil. The second experiment with AB0 chip showed a similar residual oil fraction after water flooding (1PV). However, in this case the CDG fluid without cross-linker did not decrease the residual oil saturation.

The mechanisms through which the polymer improves the sweep efficiency include the decrease of mobility ratio and diversion of injected water to places of lower permeability. Whereas CDG behavior vary according to the pressure gradient as they flow like a viscous solution at high pressure then after time has passed they can block high permeability channels [9].

Furthermore, the increased recovery was attributed to the improvement in the microscopic displacement of the flood [27]. However, further studies on the influence of CDG concentration are needed to evaluate the optimum CDG concentration.

The efficient oil displacement achieved by CDG mixture with crosslinking agent (experiment number 2) is in agreement with previous studies performed in Loma Alta Sur field. The authors suggested that the enhanced properties of CDG over uncrosslinked polymer are particularly useful in heretogenous formations, where non-uniform rock results in an uneven flood front and incomplete volumetric sweep. In this type of rock, uncrosslinked polymer is not strong enough to overcome these adverse effects [10]. Besides, ALZATE [9] determined that CDG system increments viscosity compared to polymer when crosslinking agent is added.

\section{CONCLUSIONS}

The architecture that reflects the desired residual oil saturation corresponds to "AD0", this design has two permeability zones that entrap disconnected oil ganglia. Therefore, it represents a promising model for residual oil displacement assays. Poral volumes of CDG and polymer were quantified by digital image analysis and it was observed that CDG recovered 10.2\% of additional oil (from 33.1 to 22.9\%) when 0.67 PV was injected whereas polymer recovered $26.6 \%$ of additional oil (from 32 to $5.4 \%$ ) using 0.7 PV. In addition, it was determined that CDG mixture with crosslinker agent decreased the residual oil saturation more than the CDG mixture without crosslinker agent implying significant potential oil recovery enhancement.

\section{ACKNOWLEDGMENTS}

The authors thank the financial support from ANPCyT (PICT2013-0840, PICT-2014-3722), CONICET PIP (114-2011-01-00139). We would also like to thank to INTI for the cleanroom facilities.

\section{BIBLIOGRAPHY}

[1] SORBIE, K.S., Introduction to Polymer flooding, "Polymer-Improved Oil Recovery", 1 ed., USA and Canada: CRC Press, Inc Boca Raton, Florida, 1991.

[2] NILSSON, M.A., KULKARNI, R., GERBERICH, L., et al., "Effect of fluid rheology on enhanced oil recovery in a microfluidic sandstone device”, Journal of Non-Newtonian Fluid Mechanics, v. 202, n. 9, pp. 112-119, Oct. 2013.

[3] DONG, X., LIU, H., HOU, J., et al., "Multi-thermal fluid assisted gravity drainage process: A new improved-oil-recovery technique for thick heavy oil reservoir", Journal of Petroleum Science and Engineering, v. 133, n. 1, pp. 1-11, May. 2015.

[4] RYAZANOV, A.V., SORBIE, K.S., VAN DIJKE, M.I.J. "Structure of residual oil as a function of wettability using pore-network modeling”, Advances in Water Resources, v. 63, n.2, pp. 11-21, Jan. 2004.

[5] FENG, G., YU, M. "Characterization of pore volume of cumulative water injection distribution", Petroleum, v. 1, n. 2, pp. 158-163, Jun. 2015.

[6] CHERAGHIAN, G., HENDRANINGRAT, L. "A review on applications of nanotechnology in the enhanced oil recovery part B: effects of nanoparticles on flooding”, International Nano Letters, v. 6, n. 1, pp. 110, Mar. 2015. 
[7] CONN, C.A., MA, K., HIRASAKI, G.J., et al., "Visualizing oil displacement with foam in a microfluidic device with permeability contrast", Lab on a Chip, v. 14, n. 20, pp. 3968-3977, Jul. 2014.

[8] QUENNOUZ, N., RYBA, M., ARGILLIER, J.F., et al., "Microfluidic Study of Foams Flow for Enhanced Oil Recovery (EOR)", Oil Gas Science. Technology, - Rev. IFP Energies nouvelles, v. 69, n. 3, pp. 457-466, Jul. 2014.

[9] ALZATE, D., Interpretación de los mecanismos fenomenológicos del proceso de inyección de Geles de Dispersión Coloidal (CDG) en un yacimiento de hidrocarburos., Msc Thesis, in Facultad de Minas, Escuela de Procesos y Energía., Universidad Nacional de Colombia: Medellín, Colombia, 2016.

[10] DIAZ, D., SOMARUGA, C., NORMAN, C., et al., "Colloidal Dispersion Gels Improve Oil Recovery in a Heterogeneous Argentina Waterflood", In: SPE Symposium on Improved Oil Recovery, SP113320-MS Tulsa, Oklahoma -USA. 20-23 April 2008.

[11] NEGIN, C., ALI, S., XIE, Q. "Application of nanotechnology for enhancing oil recovery- a review", Petroleum, v. 2, n. 4, pp.324-333, Oct. 2016.

[12] GRATE, J.W., ZHANG, C., WIETSMA, T.W., et al., "A note on the visualization of wetting film structures and a nonwetting immiscible fluid in a pore network micromodel using a solvatochromic dye", Water Resources Research, v. 46, n. 11, pp. w11602-w11608, Nov. 2010.

[13] LI, R., JIANG, P., GAO, C., et al., "Experimental Investigation of Silica-based Nanofluid Enhanced Oil Recovery: The Effect of Wettability Alteration", Energy \& Fuels, v. 31, n. 1, pp. 188-197, Dec. 2016.

[14] SAYEGH, S.G., D.B. FISHER. "Enhanced Oil Recovery by CO Flooding in Homogeneous and Heterogeneous 2D Micromodels", Journal of Canadian Petroleum Technology, v. 48, n. 8, pp. 30-36, Aug. 2009.

[15] GHAZANFARI, M.H., RASHTCHIAN, D., KHARAT, R., et al., "Capillary Pressure Estimation Using Statistical Pore Size Functions", Chemical Engineering \& Technology, v. 30, n. 7, pp. 1521-4125, Jul. 2007.

[16] MCDOUGALL, S.R., MACKAY, E.J. "The Impact of Pressure-Dependent Interfacial Tension and Buoyancy Forces Upon Pressure Depletion in Virgin Hydrocarbon Reservoirs", Chemical Engineering Research and Design, v. 76, n. 5, pp. 553-561, Jul. 1998.

[17] KAMARI, E., RASHTCHIAN, D., SHADIZADEH, S.R. "Immiscible Displacement of a Wetting Fluid by a Non-wetting One at High Capillary Number in a Micro-model Containing a Single Fracture", Transport in Porous Media, v. 94, n. 1, pp. 289-301, Aug. 2012.

[18] ISIKSACAN, Z., GULER, M.T., AYDOGDU, B., et al., "Rapid fabrication of microfluidic PDMS devices from reusable PDMS molds using laser ablation", Journal of Micromechanics and Microengineering, v. 26, n. 3, pp. 35008-35016, Feb. 2016.

[19] TANG, M., WANG, G., KONG, S., et al., "A Review of Biomedical Centrifugal Microfluidic Platforms", Micromachines, v. 7, n. 2, pp. 26-55, Feb. 2016.

[20] MCDONALD, J.C., WHITESIDES, G.M. "Poly(dimethylsiloxane) as a Material for Fabricating Microfluidic Devices", Accounts of Chemical Research, v. 35, n. 7, pp. 491-499, Apr. 2002.

[21] ANDERSON, W.G., "Wettability Literature Survey Part 5: The Effects of Wettability on Relative Permeability", Journal of Petroleum Techonology, v.39, n. 11, pp.1453-1468, Oct. 1987.

[22] MOHANTY, K.K., H.T. DAVIS, AND L.E. SCRIVEN, "Physics of Oil Entrapment in Water-Wet Rock", SPE Resorvoir Egineering, v. 2, n. 01, pp.113-128, Feb. 1987.

[23] AUS-e-TUTE. CHEMISTRY TUTORIAL: SURFACE TENSION.

http://www.ausetute.com.au/index.html - .WDtazM7GyAY. Accessed on October 2016

[24] VALVATNE, P.H., Predictive pore-scale modelling of multiphase flow, Ph.D Thesis, in Department of Earth Science and Engineering., Imperial College London: United Kingdom, 2004.

[25] SCHNEIDER, M., Wettability Patterning in Microfluidic Systems and Applications in the Petroleum Industry, Ph.D. Thesis, in Laboratoire Microfluidique, MEMS, Nanostructures (MMN), Université Pierre et Marie Curie - Paris VI, 2011.

[26] ANDERSON, W.G., "Wettability Literature Survey-Part 6: The Effects of Wettability on Waterflooding", Journal of Petroleum Technology, v. 39, n.12, pp.1605-1622, Dec. 1987.

[27] SKAUGE, T., SPILDO, K., SKAUGE, A., "Nano-sized Particles For EOR", SPE Symposium on Improved Oil Recovery, Tulsa, SP-129933-MS, Oklahoma - USA. 24-28 April 2010. 